\title{
ISOMORPHISMS OF TREES
}

\author{
FRANTISEK FRANEK
}

\begin{abstract}
Let $\kappa, \lambda$ be cardinals, $\kappa \geq \aleph_{1}$ and regular, and $2 \leq \lambda \leq \kappa$. If $\kappa>\aleph_{1}$ and $\lambda<\kappa$, and if there is a $\kappa$-Suslin ( $\kappa$-Aronszajn, $\kappa$-Kurepa) tree, then there are $2^{\kappa}$ normal $\lambda$-ary rigid nonisomorphic $\kappa$-Suslin ( $\kappa$-Aronszajn, $\kappa$-Kurepa) trees. If there is a Suslin (Aronszajn, Kurepa) tree, then there is a normal rigid Suslin (Aronszajn, Kurepa) tree. If there is a $\kappa$-Canadian tree, then there are $2^{\kappa}$ normal $\lambda$-ary rigid nonisomorphic $\kappa$-Canadian trees.
\end{abstract}

Introduction. The number of automorphisms of a given tree and the number of nonisomorphic trees of the same type have been investigated in several papers. It is known that

(1) it is consistent with ZFC that (a) there is a rigid Suslin tree; (b) there is a Suslin tree with $2^{\aleph_{0}}$ automorphism; (c) there is a Suslin tree with $2^{\aleph_{1}}$ automorphisms;

(2) the same as above for Kurepa trees;

(3) it is true in ZFC that (a) there are $2^{\aleph_{1}}$ nonisomorphic totally rigid Aronszajn trees; (b) there is an Aronszajn tree with $2^{\aleph_{0}}$ automorphisms; (c) there is an Aronszajn tree with $2^{\aleph_{1}}$ automorphisms.

These results can be found in $\left[\mathbf{G S}, \mathbf{J}_{2}, \mathbf{J}_{3}, \mathbf{J}_{4}, \mathbf{D}\right.$ and $\left.\mathbf{T}\right]$. We shall prove that for $\kappa$-trees for $\kappa$ regular and $\kappa>\aleph_{1}$ the situation is uniform, i.e. we shall prove that if there is a normal $\kappa$-tree then there are $2^{\kappa}$ nonisomorphic rigid normal trees of the "same type". For trees of height $\kappa$ with levels of size $\leq \kappa$ it will work even when $\kappa \geq \aleph_{1}$. For details see $[\mathbf{F}]$.

Notation and definitions. We shall use standard set-theoretical notation as possible. For all basic definitions about trees, see $\left[\mathbf{J}_{1}\right.$ and $\left.\mathbf{J}_{2}\right]$. If $\langle T, \leq\rangle$ is a tree, $x \in T$, then $o(x)$ is the order type of $x$. $T_{\alpha}$ denotes the $\alpha$ th level of $T$, i.e. the set of all nodes of $T$ of the order type $\alpha . h(T)$ denotes the height of $T$. A tree $\langle T, \leq\rangle$ is normal iff

(1) every $x \in T$ has at least two immediate successors;

(2) if $\alpha \in h(T)$ is limit and $x \neq y \in T_{\alpha}$, then there are $\beta<\alpha$ and $z_{x} \neq z_{y} \in T_{\beta}$ so that $z_{x}<x$ and $z_{y}<y$;

(3) every $x \in T$ has a successor on every higher level of $T$.

$T$ is a $\lambda$-ary tree ( $\lambda$ a cardinal), if every $x \in T$ has exactly $\lambda$ immediate successors.

Note. If $\langle T, \leq\rangle$ is a normal tree, then there is a unique (up to isomorphism) complete Boolean algebra $B$ with the canonical ordering $\leq$ so that $T \subset B$ and $\leq^{\prime} \mid T=\leq^{-1}$ and $T$ is a dense subset of $B$. If $h(T)$ is a limit ordinal, then $B$ is

Received by the editors December 28, 1984 .

1980 Mathematics Subject Classification. Primary 04A20; Secondary 06E99. 
atomless. If $T$ is $\nu$-ary, then every element of $B-\left\{O_{B}\right\}$ can be partitioned into $\nu$ mutually disjoint elements of $B-\left\{O_{B}\right\}$.

Let $B$ be a complete Boolean algebra with the canonical ordering $\leq^{\prime}$. Let $\langle T, \leq\rangle$ be a normal $\nu$-ary tree dense in $B$. $B(\nu, T)$ is the set of all elements of $B$ that are a union of an antichain of $T$ of size $\nu$. If $x \in B-\left\{O_{B}\right\}$, then $B \mid x=\{y \in B: y \leq x\}=$ $\{x \wedge y: y \in B\}$ (note that $B \mid x$ with the restricted operations is again a complete Boolean algebra and $O_{B \mid x}=O_{B}$ and $\left.1_{B \mid x}=x\right) . B(\nu, T)|x=B(\nu, T) \cap B| x$. A tree $\left\langle R, \leq_{R}\right\rangle$ is built in $B(\nu, T) \mid x$ if $\mathbf{R} \subset B(\nu, T) \mid x, \overline{\leq^{\prime} \mid R=} \leq_{R}^{-1}$ and $\sum R_{\alpha}=x$ for every $\alpha<h(R)$.

$T$ is a $\kappa$-Canadian tree if $T$ has height $\kappa$, all levels of size $\leq \kappa$, and at least $\kappa^{+}$cofinal branches (i.e. branches of the length $h(T)$ ). A Canadian tree is an $\aleph_{1^{-}}$ Canadian tree. A Canadian tree is nice if there is a cofinal branch passing through every node.

\section{Preliminaries.}

LEMMA 1. Let $\kappa \geq \aleph_{1}$, be regular. Then there are mutually disjoint sets $K_{1}, K_{2}, K_{3}$ of size $\kappa$ and a function $h$ so that

(1) $K_{1} \cup K_{2} \cup K_{3}=\{\lambda \in \kappa: \lambda$ is limit $\}$;

(2) $\left(\forall \gamma \in K_{1} \cup K_{2}\right)\left(\gamma+\omega \in K_{3}\right)$;

(3) $h: \kappa \times \kappa \times \kappa \rightarrow K_{1}$ is a bijection such that $h(\alpha, \beta, \gamma)>\alpha$ for all $\alpha, \beta, \gamma \in \kappa$.

ProOF. Left to the reader.

LEMMA 2. Let $\kappa, \lambda$ and $\nu$ be cardinals, $\kappa \geq \aleph_{1}$ and regular, $2 \leq \lambda \leq \kappa$ and $\nu=\max \left\{\lambda, \aleph_{1}\right\}$. Then there are two nonisomorphic normal $\lambda$-ary trees $R^{b}$ and $R^{w}$ of height $(\omega+1)$ such that all their levels are of size $\leq \lambda \cdot \aleph_{0}$ and $\left|R_{\omega}^{w}\right|=\nu$.

PROOF. (a) Case when $2 \leq \lambda \leq \aleph_{0}$. Let $R_{n}^{b}=R_{n}^{w}={ }^{n} \lambda$ for every $n \in \omega$. For every $f \in{ }^{<\omega} \lambda$ choose some $g_{f} \in{ }^{\omega} \lambda$ so that $g_{f} \mid \operatorname{dom}(f)=f$. Let $R_{\omega}^{b}=\left\{g_{f}: f \in\right.$ $\left.{ }^{<\omega} \lambda\right\}$. Then $R_{\omega}^{b} \subset{ }^{\omega} \lambda$ and $\left|R_{\omega}^{b}\right|=\aleph_{0}$. Since $\left|{ }^{\omega} \lambda\right| \geq \aleph_{1}$, there is some $R_{\omega}^{w} \subset{ }^{\omega} \lambda$ so that $\left|R_{\omega}^{w}\right|=\aleph_{1}$ and $R_{\omega}^{b} \subset R_{\omega}^{w}$. Let $R^{b}=\bigcup\left\{R_{\alpha}^{b}: \alpha \leq \omega\right\}$ and $R^{w}=\bigcup\left\{R_{\alpha}^{w}: \alpha \leq \omega\right\}$. For $f, g \in R^{b}\left(R^{w}\right)$ define $f \geq g$ iff $f \supset g$. Then $R^{b}, R^{w}$ are normal $\lambda$-ary trees of height $(\omega+1)$ with all levels countable, but $\left|R_{\omega}^{w}\right|=\aleph_{1}=\nu . R^{b}$ and $R^{w}$ are not isomorphic for $\left|R_{\omega}^{b}\right| \neq\left|R_{\omega}^{w}\right|$.

(b)Case when $\aleph_{1} \leq \lambda<\kappa$. If $f \in{ }^{\omega} \lambda$, then $f \equiv 0$ iff $(\exists n \in \omega)(\forall m \in \omega)$ $(m \geq n \Rightarrow f(m)=0)$. Let $R_{\alpha}^{b}=\left\{f \mid \alpha: f \in{ }^{\omega} \lambda \& f \equiv 0\right\}$, for all $\alpha \leq \omega$. Let $R^{b}=$ $\bigcup\left\{R_{\alpha}^{b}: \alpha \leq \omega\right\}$. For $f, g \in R^{b}$ define $f \geq g$ iff $f \supset g$. Then $\left\langle R^{b}, \leq\right\rangle$ is a normal $\lambda$-ary tree of height $(\omega+1)$ with all levels of size $\leq \lambda$. Let $T$ be a normal binary tree of height $(\omega+1)$ so that $\left|T_{\omega}\right|=\aleph_{0}$. Let $R^{w}$ be a product of $\lambda$ copies of $T$. Then $R^{w}$ is a normal $\lambda$-ary tree of height $(\omega+1)$ with all levels of size $\lambda . R^{b}$ and $R^{w}$ are not isomorphic, for $R^{w}$ contains a copy of a binary tree, while $R^{w}$ does not.

LEMMA 3. Let $\kappa, \lambda, \nu$ be cardinals, $\kappa \geq \aleph_{1}$ and regular, $2 \leq \lambda \leq \kappa$ and $\nu=$ $\max \left\{\lambda, \aleph_{1}\right\}$. Let $T$ be a normal $\nu$-ary tree of height $\kappa$ dense in a complete Boolean algebra $B$. Let $x \in B-\left\{O_{B}\right\}$. Then there are two nonisomorphic $\lambda$-ary normal trees $T^{w}$ and $T^{b}$ of height $(\omega+1)$ with all levels of size $\leq \lambda \cdot \aleph_{0}$ built in $B(\nu, T) \mid x$ and so that $T_{\omega}^{w} \subset T$.

Proof. (HINT) Build $T^{w}$ in $B(\nu, T) \mid x$ as an isomorphic copy of $R^{w}$ and $T^{b}$ as an isomorphic copy of $R^{b}\left(R^{w}, R^{b}\right.$ from Lemma 2$)$, using the fact that every 
nonzero element of $B(\nu, T) \mid x$ can be partitioned into $\nu$ mutually disjoint nonzero elements of $B(\nu, T)$.

\section{Results.}

THEOREM 4. Let $\kappa, \lambda, \nu$ be cardinals, $\kappa \geq \aleph_{1}$ and regular, $2 \leq \lambda \leq \kappa$ and $\nu=\max \left\{\lambda, \aleph_{1}\right\}$. Let (I) $\kappa>\aleph_{1}$ and $T$ be a normal $\nu$-ary $\kappa$-tree, or (II) $T$ be a normal nice $\nu$-ary $\kappa$-Canadian tree. Let $T$ be dense in a complete Boolean algebra $B$ with the canonical ordering $\leq$. Then there is a sequence $\left\langle R^{\theta}: \theta \in 2^{\kappa}\right\rangle$ so that

(1) $R^{\theta}$ with $\leq^{-1}$ is a rigid normal $\lambda$-ary tree of height $\kappa$ with as many cofinal branches as $T$; in case (I), $R^{\theta}$ is a $\kappa$-tree, in case (II), $R^{\theta}$ is a $\kappa$-Canadian tree;

(2) $R^{\theta}$ is dense in $B$

(3) if $\theta_{1} \neq \theta_{2} \in 2^{\kappa}$, then $R^{\theta_{1}}$ and $R^{\theta_{2}}$ are not isomorphic.

Proof. Let $K_{1}, K_{2}, K_{3}$ and $h$ be as in Lemma 1. Let $P\left(K_{2}\right)=\left\{X_{\theta}: \theta \in 2^{\kappa}\right\}$. Fix $\theta \in 2^{\kappa}$. We shall describe the construction of $R^{\theta}$. For simplicity we shall omit the superscript $\theta$. Let $X=X_{\theta}$.

By transfinite recursion we shall construct two sequences $\left\langle R_{\alpha}: \alpha<\kappa\right\rangle$ and $\left\langle f_{\alpha}: \alpha \in \kappa\right\rangle$ so that, for any $\alpha<\kappa$,

(i) $R_{\alpha}$ is a partition of $1_{B}$ of size $<\kappa$ (in case (I)) or $\leq \kappa$ (in case (II));

(ii) $R_{\alpha} \subset B(\nu, T)$;

(iii) $R_{\alpha+\omega} \subset T$ whenever $\alpha \in K_{3}$;

(iv) $R \mid \alpha+1=\bigcup\left\{R_{\beta}\right.$ : $\left.\beta \leq \alpha\right\}$ with $\leq^{-1}$ is a normal $\lambda$-ary tree of height $(\alpha+1)$;

(v) $f_{\alpha}$ is an enumeration of $R_{\alpha}$ with $\operatorname{rng}\left(f_{\alpha}\right) \subset \kappa$.

The construction will be described in three steps.

(A) $R_{0}=\left\{1_{B}\right\}$ and $f_{0}=\left\{\left\langle 1_{B}, 0\right\rangle\right\}$, i.e. $f_{0}\left(1_{B}\right)=0$.

(B) Suppose that we have already constructed $\left\langle R_{\alpha}: \alpha \leq \beta\right\rangle$ and $\left\langle f_{\alpha}: \alpha \leq \beta\right\rangle$ for $\beta<\kappa$ limit or $\beta=0$. We shall describe the construction of $R_{\beta+1}, \ldots, R_{\beta+\omega}$ and $f_{\beta+1}, \ldots, f_{\beta+\omega}$. First we shall color the elements of $R_{\beta}$.

(B1) Case when $\beta \in K_{1}$. Then $\beta=h(\gamma, \rho, \delta)$ for some $\gamma, \rho, \delta \in \kappa$ and $\gamma<\beta$. If $\rho, \delta \in \operatorname{mg}\left(f_{\gamma}\right)$ and if there exists a nontrivial automorphism of $R \mid \beta+1$ mapping $f_{\gamma}^{-1}(\rho)$ onto $f_{\gamma}^{-1}(\delta)$, then every $x \in R_{\beta}$ so that $x<f_{\gamma}^{-1}(\rho)$ is colored "black"; the rest are colored "white". Otherwise, all elements of $R_{\beta}$ are colored "white".

(B2) Case when $\beta \in K_{2}$. If $\beta \in X$ (the fixed subset of $K_{2}$ ), then all elements of $R_{\beta}$ are colored "white"; otherwise all elements of $R_{\beta}$ are colored "black".

(B3) Case when $\beta \in K_{3}$ or $\beta=0$. Then all elements of $R_{\beta}$ are colored "white".

Now, after the coloring, we can construct $R_{\beta+1}, \ldots, R_{\beta+\omega}$ and $f_{\beta+1}, \ldots, f_{\beta+\omega}$. If $x \in R_{\beta}$ was colored "white", let $\left\langle R_{x, \alpha}: \alpha \leq \omega\right\rangle$ be the tree $T^{w}$ built in $B(\nu, T) \mid x$, otherwise (if $x$ is colored "black") let it be the tree $T^{b}$ built in $B(\nu, T) \mid x\left(T^{w}, T^{b}\right.$ from Lemma 3). For all $\alpha \leq \omega$ define $R_{\beta+\alpha}=\bigcup\left\{R_{x, \alpha}: x \in R_{\beta}\right\}$ and define $f_{\beta+\alpha}$ as an enumeration of $R_{\beta+\alpha}$ with $\operatorname{rng}\left(f_{\beta+\alpha}\right) \subset \kappa$.

It is left to the reader to check that (i)-(v) hold. When proving that (iii) holds, realize that, for any $x \in R_{\beta}, R_{x, \omega} \subset T$ (by Lemma 3) and so $R_{\beta+\omega} \subset T$.

(C) Suppose that we have already constructed $\left\langle R_{\alpha}: \alpha<\beta\right\rangle$ and $\left\langle f_{\alpha}: \alpha<\beta\right\rangle$ for $\beta<\kappa$ limit. First note that there is no $\gamma$ so that $\beta=\gamma+\omega$. If so, there is $\gamma$ limit or $\gamma=0$ so that $\beta=\gamma+\omega$. Hence, after $R_{\gamma}$ was constructed, the levels $R_{\gamma+1}, \ldots, R_{\gamma+\omega}$ were constructed according to (B), and thus $R_{\beta}$ is already constructed, too. 
Let $\alpha<\beta$. Then $(\alpha+n \cdot \omega)<\beta$ for any $n<\omega$ and $\alpha+\omega$ is limit; hence $\alpha+\omega \in K_{1} \cup K_{2} \cup K_{3}$. If $\alpha+\omega \in K_{1} \cup K_{2}$, then $\alpha+\omega+\omega \in K_{3}$ and so $R_{\alpha+\omega+\omega+\omega} \subset T$. Define $\gamma_{\alpha}=\alpha+\omega+\omega+\omega$. If $\alpha+\omega \in K_{3}$, then $R_{\alpha+\omega+\omega} \subset T$ and thus define $\gamma_{\alpha}=\alpha+\omega+\omega$. So in any case, for every $\alpha<\beta$ there is $\gamma_{\alpha}$ so that $\alpha<\gamma_{\alpha}<\beta$ and $R_{\gamma_{\alpha}} \subset T$. Let $\left\langle\lambda_{\alpha}: \alpha<\rho\right\rangle$ be a strictly increasing sequence so that $\left\{\lambda_{\alpha}: \alpha<\rho\right\} \subset\left\{\gamma_{\alpha}: \alpha<\beta\right\}$ and $\bigcup\left\{\lambda_{\alpha}: \alpha<\rho\right\}=\bigcup\left\{\gamma_{\alpha}: \alpha<\beta\right\}=\beta$. Thus for each $\alpha<\rho, R_{\lambda_{\alpha}} \subset T$.

Let $x \in \bigcup\left\{R_{\lambda_{\alpha}}: \alpha<\rho\right\}$.

In case (I), for every $\alpha<\rho$ define $\mu(\alpha)=\sup \left\{\mu \in \kappa: R_{\lambda_{\alpha}} \cap T_{\mu} \neq \varnothing\right\}$. Since $\left|R_{\lambda_{\alpha}}\right|<\kappa$ and $\kappa$ is regular, then $\mu(\alpha)<\kappa$. Now let $\mu<\kappa$ be so that $\mu>\bigcup\{\mu(\alpha): \alpha<\rho\}$. Hence for any $\alpha<\rho, R_{\lambda_{\alpha}} \cap T_{\mu}=\varnothing$. By normality of $T$, there is $y \in T_{\mu}$ so that $y<x$ and for every $\alpha<\rho$ there is a unique $x_{\alpha} \in R_{\lambda_{\alpha}}$ so that $y<x_{\alpha} .\left\{x_{\alpha}: \alpha<\rho\right\}$ determines a cofinal branch $b_{x}$ of $R \mid \beta$ so that $x \in b_{x}$ and $\prod b_{x} \in T$.

In case (II), there is a cofinal branch $d$ in $T$ so that $x \in d$. Since $d$ intersects every $R_{\lambda_{\gamma}}, \gamma<\rho$, it determines a unique cofinal branch $b_{x}$ in $R \mid \beta$ so that $x \leq \prod b_{x}$ and $\prod b_{x} \in T$.

Define $R_{\beta}=\left\{\prod b_{x}: x \in \bigcup\left\{R_{\lambda_{\alpha}}: \alpha<\rho\right\}\right\}$. Then $R_{\beta}$ is an antichain, in case (I) $\left|R_{\beta}\right|<\kappa$ as $\left|\bigcup\left\{R_{\lambda_{\alpha}}: \alpha<\rho\right\}\right|<\kappa$, and in case (II), $\left|R_{\beta}\right| \leq \kappa$ as $\left|\bigcup\left\{R_{\lambda_{\alpha}}: \alpha<\rho\right\}\right| \leq$ $\kappa$, and $\sum R_{\beta}=1_{B}$, and $R_{\beta} \subset T$; hence $R_{\beta} \subset B(\nu, T)$. Let $f_{\beta}$ be an enumeration of $R_{\beta}$ with $\operatorname{rng}\left(f_{\beta}\right) \subset \kappa$.

It is left to the reader to check that (i) $-(\mathrm{v})$ hold.

Define $R=\bigcup\left\{R_{\alpha}: \alpha<\kappa\right\}$ and $f=\bigcup\left\{f_{\alpha}: \alpha<\kappa\right\}$. This completes the construction.

Claim 1. In case (I), $R$ is a rigid normal $\lambda$-ary $\kappa$-tree with as many cofinal branches as $T$. In case (II), $R$ is a rigid normal $\lambda$-ary $\kappa$-Canadian tree with as many cofinal branches as $T$.

From (iv) follows that $R$ is a normal $\lambda$-ary of height $\kappa$. From (i) follows that $R$ is a $\kappa$-tree (in case (I)), or a tree with levels of size $\leq \kappa$ (in case (II)).

Let us prove that $R$ is rigid. Suppose not. Then there are $\gamma<\kappa$ and a nontrivial

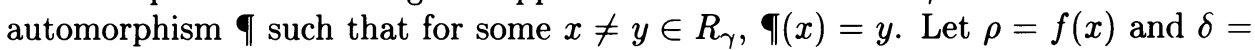
$f(y)$. Let $\beta=h(\gamma, \rho, \delta)$. Then $\beta \in K_{1}$ and $\gamma<\beta$. There are $z_{x} \neq z_{y} \in R_{\beta}$ so that $\mathbb{\Upsilon}\left(z_{x}\right)=z_{y}, z_{x} \leq x$ and $z_{y} \leq y$. Now recall how $R_{\beta+1}, \ldots, R_{\beta+\omega}$ were constructed (case (B1)). Every $z \in R_{\beta}$ so that $z<x$ was colored "black" and the rest were colored "white", for $\Phi(R \mid \beta+1)$ is a nontrivial automorphism of $R \mid \beta+1$ so that it maps $x=f^{-1}(\gamma)$ onto $y=f^{-1}(\rho)$ and $\beta=h(\gamma, \rho, \delta) . R_{\beta+\alpha}=\bigcup\left\{R_{z, \alpha}: z \in R_{\beta}\right\}$ for all $\alpha \leq \omega$. Then $\left\langle R_{z_{x}, \alpha}: \alpha \leq \omega\right\rangle$ is an isomorphic copy of $T^{w}$ built in $B(\nu, T) \mid z_{x}$ and $\left\langle R_{z_{y}, \alpha}: \alpha \leq \omega\right\rangle$ is an isomorphic copy of $T^{b}$ built in $B(\nu, T) \mid z_{y}$. Since $\mathbb{\Psi}\left(z_{x}\right)=\mathbb{\Psi}\left(z_{y}\right)$,

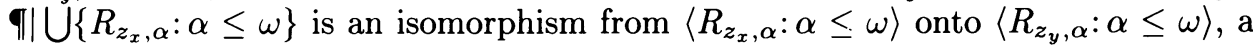
contradiction as $T^{w}$ and $T^{b}$ are not isomorphic.

Let us conclude the proof of Claim 1 by showing that $R$ has as many cofinal branches as $T$. Let $b=\langle b(\alpha): \alpha<\kappa\rangle$ be a cofinal branch of $R$. If $\alpha \in K_{3}$, then $R_{\alpha+\omega} \subset T$ by (iii). Hence $\left\langle b(\alpha+\omega): \alpha \in K_{3}\right\rangle$ is a linearly ordered cofinal subset of $T$ and determines a unique cofinal branch of $T$. Thus $T$ has at least as many cofinal branches as $R$.

Let $b$ be a cofinal branch of $T$. Then for any $\alpha \in K_{3}, R_{\alpha+\omega} \subset T$ and $\sum R_{\alpha+\omega}=$ $1_{B}$. So $R_{\alpha+\omega}$ is a maximal antichain in $T$ of size $<\kappa$. Hence $\left|b \cap R_{\alpha+\omega}\right|=1$. Then 
$\left\{y \in T:\left(\exists \alpha \in K_{3}\right)\left(\{y\}=b \cap R_{\alpha+\omega}\right)\right\}$ is a cofinal linearly ordered subset of $R$, hence it determines a unique cofinal branch in $R$. Thus $R$ has at least as many cofinal branches as $T$.

Since in case (II), $T$ was a $\kappa$-Canadian tree, $R$ is a $\kappa$-Canadian tree as well. Claim 1 is proven.

Claim 2. $R$ is dense in $B$. Let $y \in B-\left\{O_{B}\right\}$. Then there is an $x \in T$ so that $x \leq y$ for $T$ is dense in $B$. If $\alpha \in K_{3}$, then $R_{\alpha+\omega}$ is a maximal antichain in $T$, and so there is $z_{\alpha} \in R_{\beta+\omega}$ comparable with $x$. Since $\alpha_{1} \neq \alpha_{2} \in K_{3} \Rightarrow z_{\alpha_{1}} \neq z_{\alpha_{2}}$, there is $\alpha \in K_{3}$ so that $z_{\alpha} \leq x$. Hence $R$ is dense in $T$. Claim 2 is proven.

Now consider the whole sequence $\left\langle R^{\theta}: \theta \in 2^{\kappa}\right\rangle$.

Claim 3. Let $\theta_{1} \neq \theta_{2} \in 2^{\kappa}$. Then $R^{\theta_{1}}$ and $R^{\theta_{2}}$ are not isomorphic.

Assume that $\Psi: R^{\theta_{1}} \rightarrow R^{\theta_{2}}$ is an isomorphism. Since $\theta_{1} \neq \theta_{2}, X_{\theta_{1}} \neq X_{\theta_{2}}$. Without loss of generality assume that there is $\beta \in X_{\theta_{1}}-X_{\theta_{2}}$. Then $\beta \in K_{2}$. By (B2) all elements of $R_{\beta}^{\theta_{1}}$ were colored "white" for $\beta \in X_{\theta_{1}}$ and all elements of $R_{\beta}^{\theta_{2}}$ were colored "black" for $\beta \notin X_{\theta_{2}}$. Let $x \in R_{\beta}^{\theta_{1}}$. Then $\left\langle R_{x, \alpha}^{\theta_{1}}: \alpha \leq \omega\right\rangle$ is an isomorphic copy of $T^{w}$ built in $B(\nu, T) \mid x$. Let $y=\mathbb{\Upsilon}(x)$. Since $y \in R_{\beta}^{\theta_{2}},\left\langle R_{y, \alpha}^{\theta_{2}}: \alpha \leq \omega\right\rangle$ is an isomorphic copy of $T^{b}$ built in $B(\nu, T) \mid y$. Since $\mathbb{\Phi}(x)=y, \mathbb{\|} \mid\left(\bigcup\left\{R_{x, \alpha}^{\theta_{1}}: \alpha \leq \omega\right\}\right)$ is an isomorphism from $\left\langle R_{x, \alpha}^{\theta_{1}}: \alpha \leq \omega\right\rangle$ onto $\left\langle R_{y, \alpha}^{\theta_{2}}: \alpha \leq \omega\right\rangle$, a contradiction as $T^{w}$ and $T^{b}$ are not isomorphic. Claim 3 is proven.

Thus the sequence $\left\langle R^{\theta}: \theta \in 2^{\kappa}\right\rangle$ satisfies (1)-(3) (proven by Claims 1-3).

COROLlaRY 5. Let $\kappa$ and $\lambda$ be cardinals, $\kappa$ regular, and $2 \leq \lambda<\kappa$. Then

(1) if $\kappa>\aleph_{1}$ and if there is a $\kappa$-Suslin tree, then there are $2^{\kappa}$ nonisomorphic rigid normal $\lambda$-ary $\kappa$-Suslin trees;

(2) if $\kappa>\aleph_{1}$ and if there is a $\kappa$-Aronszajn tree, then there are $2^{\kappa}$ nonisomorphic rigid normal $\lambda$-ary $\kappa$-Aronszajn trees;

(3) if $\kappa>\aleph_{1}$ and if there is a $\kappa$-Kurepa, then there are $2^{\kappa}$ nonisomorphic rigid normal $\lambda$-ary $\kappa$-Kurepa trees;

(4) if $\lambda \leq \kappa, \aleph_{0} \leq \kappa$ and if there is a $\kappa$-Canadian tree, then there are $2^{\kappa}$ nonisomorphic rigid normal $\lambda$-ary $\kappa$-Canadian trees.

PROOF. (SKETCH) Let $\nu=\max \left\{\lambda, \aleph_{1}\right\}$. If there is a tree $\hat{T}$ of certain type, then there is a normal $\nu$-ary tree $T$ of the same type. In case $\hat{T}$ was $\kappa$-Canadian, there is a nice $T$. Let $T$ be dense in a complete Boolean algebra $B$. If $T$ is $\kappa$-Suslin, then $B$ satisfies the $\kappa$-c.c. (i.e. every chain or antichain in $B$ has size $<\kappa$ ) and so every $\kappa$-tree built in $B$ must be $\kappa$-Suslin. Let $\left\langle R^{\theta}: \theta \in 2^{\kappa}\right\rangle$ be the sequence from Theorem 4. Since the type of the tree we are dealing with is determined either by the number of cofinal branches or by the size of antichains and chains, each $R^{\theta}$ is tree of the same type as $T$.

REMARK. Unfortunately, the method of Theorem 4 cannot be used for $\kappa=\aleph_{1}$ and trees with countable levels, for $T^{w}$ and $T^{b}$ are isomorphic if they have all levels countable. Nevertheless, the technique of complete Boolean algebras generated by normal trees could be used to obtain some (although not too interesting) rigid $\aleph_{1}$-trees.

By induction construct a normal trees $R^{w}$ of height $(\omega+1)$ with all levels of countable size and so that every level is enumerated and if $x$ is the $n$th element of the $m$ th level, then $x$ has exactly $n$ immediate successors at the $(m+1)$ th level. 
Such a tree is rigid. If $T$ is a normal $\aleph_{0}$-ary $\aleph_{1}$-tree dense in a complete Boolean algebra $B$ and $x \in B-\left\{O_{B}\right\}$, then there is a rigid normal tree $T^{w}$ of height $(\omega+1)$ with all levels of countable size built in $B\left(\aleph_{1}, T\right)$ and so that $T_{\omega}^{w} \subset T$ (it is an isomorphic copy of $R^{w}$ ). If a tree $R$ is constructed in the same way as $R^{\theta}$ was constructed in Theorem 4 with the exception that all elements are always colored white, then it will prove the following theorem:

THEOREM 6. Let $T$ be a normal $\aleph_{0}$-ary $\aleph_{1}$-tree dense in a complete Boolean algebra $B$. Then there is a normal rigid $\aleph_{1}$-tree $R$ dense in $B$ and with as many cofinal branches as $T$.

COROLlaRY 7. Let there be a Suslin (Aronszajn, Kurepa) tree. Then there is a normal rigid Suslin (Aronszajn, Kurepa) tree.

\section{REFERENCES}

[D] K. J. Devlin and H. Johnsbraten, The Souslin problem, Lecture Notes in Math., vol. 405, Springer-Verlag, Berlin and New York, 1974.

[G] H. Gaifman and E. P. Specker, Isomorphism types of trees, Proc. Amer. Math. Soc. 15 (1964), 1-7.

[F] F. Franek, Some results about saturated ideals and about isomorphisms of $\kappa$-trees, Ph. D. Thesis, University of Toronto, 1983.

[J $\left.\mathbf{J}_{1}\right]$ T. J. Jech, Set theory, Academic Press, New York, 1980.

$\left[\mathbf{J}_{2}\right]$, Trees, J. Symbolic Logic 36 (1971), 1-14.

$\left[\mathbf{J}_{3}\right] \_$, Automorphisms of $\omega_{1}$-trees, Trans. Amer. Math. Soc. 173 (1972), 57-70.

[J $\left.\mathbf{J}_{4}\right]$, Simple complete Boolean algebra, Israel J. Math. 18 (1974).

[T] S. B. Todorcevic, Rigid Aronszajn trees, Publ. Inst. Math. (Beograd) (N. S. ) 27 (1980).

Department of Mathematics and Computer Science, Dartmouth College, HANOVER, NEW HAMPSHIRE 03755 10027

Current address: Department of Computer Science, Columbia University, New York, New York 